\title{
The AURA Observatory in Chile-part of the IAU/UNESCO Extended Case Study
}

\author{
Malcolm G. Smith ${ }^{1}$, R. Chris Smith ${ }^{2}$ and Pedro Sanhueza ${ }^{3}$ \\ ${ }^{1} \mathrm{NOAO} / \mathrm{CTIO}$, \\ Avenida Juan Cisternas 1600, La Serena, Chile \\ email: msmith@ctio.noao.edu \\ ${ }^{2}$ AURA Observatory in Chile, \\ Avenida Juan Cisternas 1600, La Serena, Chile \\ email: csmith@aura-o.aura-astronomy.org \\ ${ }^{3} \mathrm{OPCC}$, \\ Avenida Juan Cisternas 1606, La Serena, Chile \\ email: psanhueza2007@gmail.com
}

Brief report. The Extended Case Study for AURA-O as a "Window to the Universe" (http://www2.astronomicalheritage.net/index.php/show-entity?identity=000059\&idsubentity $=005)$ was prepared in the context of supporting the desire to preserve humanity's scientific/cultural heritage of outstanding, high-mountain, ground-based, observatory sites developed over the period 1870-2000.

AURA-O includes the Cerro Tololo InterAmerican Observatory (CTIO) established in 1962 as the first of the major international observatories to be installed in Chile. The future of AURA-O now includes the Large Synoptic Survey Telescope (LSST). This Extended Case Study has provided the context for the development of possible initiatives to protect a variety of sites in Chile (e.g. Tololo, Pachón, La Silla, Las Campanas, Paranal, Armazones and Chajnantor) for their historical and scientific value to humanity. The dark skies and ideal weather patterns of northern Chile, along with its location in the southern hemisphere, have made this area of the world a major centre for astronomical facilities.

While this talk touched on the importance of dark skies as part of the Windows to the Universe concept, others will discuss the current status and future plans (of the Chilean Government and the observatories) for protecting the dark skies of northern Chile. 